\title{
Managing Congestion in Vehicular Networks Using Tabu Search
}

\author{
Muhammad Ishaq ${ }^{1}$, Mazhar Hussain Malik ${ }^{2}$, and Mehmet Emin Aydin ${ }^{3}$ \\ 1 Institute of Southern Punjab, Multan, Pakistan \\ muhammadishaqkhoja@gmail.com \\ 2 Dept. of Computing, \\ Global College of Engineering and Technology, Muscat, Oman \\ mazhar@gcet .edu.om \\ 3 Dept. of Computer Science and Creative Technology, \\ University of West of England, Bristol, UK \\ mehmet.aydin@uwe.ac.uk
}

\begin{abstract}
In this era of communication, exponentially growing networks bring a lot of challenges to address for smoother network functionalities. Among them is efficiency in handling packet traffic to avoid and control congestion. A particular case is applicable to Vehicular Ad-hoc Networks, which are known with unbalanced resource utilisation, communication overheads, high transmission delay and least transmission capacity. This paper aims to minimise the delay and jitter for enhancing the Quality of Service (QoS) in Vehicular Adhoc Networks (VANET) using tabu search algorithm with multi-channel allocation capability. We proposed a scheme that prioritises each message considering the basis of message type or its substances, such as crisis, reference point, and administration oriented etc., and uses tabu search for scheduling the transmission of queued messages in order to enhance the efficiency, security, and durability of VANET. A comprehensive simulation is conducted to validate the proposed scheme and to evaluate the performances in comparison with other state-of-the-art approaches.
\end{abstract}

Keywords: Congestion Control - Tabu Search · Dynamic message scheduling · Priorities assignment messages · VANETs Highways and Urban environment .

\section{Introduction}

Vehicular Adhoc Networks (VANETs) are particular implementation of Mobile Adhoc Networks (MANETs), which involves many network qualities and characteristics of MANETs alongside novel attributes including frequent changes in topologies. Secure and reliable communication with MANETs (and subsequently VANETs) remains as one of the main challenges being addressed through optimisation; by minimising delay and maximising the throughput of the network. In particular case of VANETs, communication can easily be blocked/disrupted due to not-well controlled message traffic and the channels can be saturated by collisions in the networks. Highly dynamically changing circumstances of VANETs 
such as fast mobility and frequent changes in network topology make congestion control problem in VANETs very attractive for investigation.

The clients of Vehicular Networks can be aware of unfavorable circumstances caused by vehicular correspondences and transmitting the data about encompassing situations $[1,2,19]$. Congestion happens in the channels when these channels face a dense vehicular saturation that is contending to access the channel. The proportional increase in the bandwidth usage due to growing the vehicle density causes congestion and may lead to crashes. The inevitable consequences of congestion include delay in transmission and losses in packet delivery, which affects the QoS and the efficiency of the Vehicular Networks. Therefore, an efficient QoS for VANETs is crucially required and can only be obtained by implementing a reliable and more secure networks $[4,6,8]$.

Congestion control problem can be solved through studying control parameters in VANETs. Prospective approaches include tuning the transmission rate or applying some control mechanism on communication and deciding the size of conflict window. These would also encompass checking Arbitration InterFrame Spacing (AIFS) or studying prioritisation strategies on beacons. Inefficient congestion control strategies escalate to emerging severe issues including high transmission delay, unbalanced resource utilisation, less efficient bandwidth utilisation, correspondence overhead, and processing overheads are some example problems that are faced during congestion management strategies [6]. This study focuses on optimising the congestion control strategy to help minimise the packet loss and delay in transmission through optimally prioritising messages in the queues and subsequently reduce the channel loads.

In this paper, saturating traffic circumstances of VANETs are considered in managing message traffic and congestion control via optimising message routes using tabu search as one of prominent metaheuristic approaches. tabu search has a success-proven record in problem solving and is adaptable to be used for instant decision making $[13,5]$. In the rest of the paper, VANET architecture and messaging infrastructure is introduced in Section 2, related works are discussed in Section 3, details of proposed approach in solving congestion control problem in Section 4 and experimental results are demonstrated and discussed in Section 5 following by conclusions in Section 6 .

\section{System Model}

The system model of this problem appears to be VANET architecture composing the main characteristics of a typical VANET implementation. There are three incorporating sub-domains of VANETs; in-vehicle domain, ad-hoc domain, and infrastructure space. In-vehicle domain requires every vehicle to be furnished with On-Board Units (OBUs) to exploit them during short range remote correspondence for security and non-critical safety transmissions. The combination of OBUs and Road side Units (RSUs) compose Ad-hoc domain, which is to deal with inter-vehicle correspondences, where OBUs transmission can be in the form of single-hop or multi-hop transmission [24]. Infrastructure domain consist of RSUs and Hotspots (HS) while OBUs can utilize mobile systems architecture. 
These architecture associated with a number of different networks such as GPRS, GSM, UMTS, or any other like WiMAX as VANET architecture is displayed in Fig. 1.

The goal for In-vehicle domain is to facilitate inter-vehicle communication to share information such as position and speed to prevent accidents. Depending on the technology, vehicles may simply receive a risk warning of an accident or vehicle itself can take actions such as slow down or breaking. In the case of only one vehicle is in the communication range then uni-cast communication will be established otherwise it become a broadcast for that particular region. In the case of Adhoc domain where vehicles are communicating with RSU or vice-versa. Vehicle to RSU have one to one communication which include information such as speed, velocity and load on road and from RSU to Vehicles can have multi-cast or broadcast communication which includes information such as load on network and delay factors in that particular region. Infrastructure domain establishes communication between different RSU with server using Internet. Communication between the RSU to server can be uni-cast, multi-cast or broadcast to pass information depending upon situation.

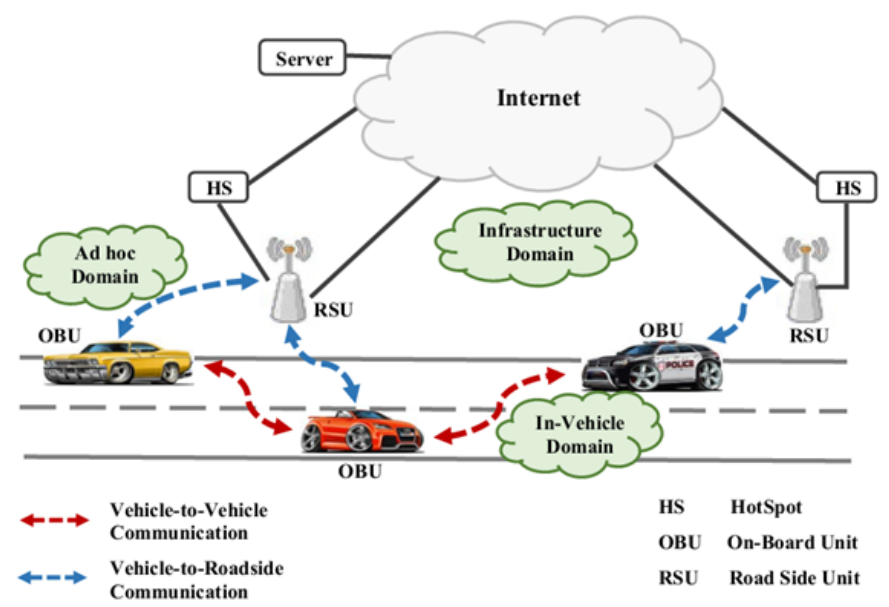

Fig. 1: VANET Architecture

\section{Related Works}

Since a decade, a substantial amount of research has been done to handle congestion in VANETs through a variety of congestion control schemes, which appear to have significant limitations due to various shortcomings including frequent changes in topologies. In the following section, relevant recent research in this regard is overviewed.

Research reported in [14] addresses vehicular safety by introducing two congestion control approaches which work at MAC layer transmission queues including measurement and even driven detection. In case of event driven detection, congestion control is launched when safety message are generated. In order to 
detect congestion in measurement based mechanism, each device, after a specific time period, senses channel usage level to check over utilization. Once congestion is detected, MAC queue manipulation mechanism handle it. Carrier Sense Multiple Access with Collision Avoidance (CSMA/CA) is used in VANETs and it was defined by IEEE $802.11 \mathrm{p}$ [12]. CSMA/CA uses default gateway strategy with exponential back-off mechanism to control congestion.

Rate adjustment scheme was proposed in [15] to ensure fair resource assignment in the networks. Mechanism measures traffic to compute channel utilization and help identify number of vehicles in the surrounding areas. However, this scheme leads to poor bandwidth management. Hannak demonstrates in [12] that the detected congestion control schemes categories as proactive and reactive. Reactive schemes obtain feedbacks from the networks to manage load, use channel states and react benchmarking with previously used pre-defined thresholds. On the other hand, proactive schemes are based on future prediction of traffic.

In $[16,17]$, researchers investigate the impact of queue freezing schemes on VANETs performance, which are caused by stopping queue once event driven safety messages detected. VANETs interference was addressed by $[18,20]$. Authors of [23] introduce a mechanism to handle congestion in short range communication devices, while [21] reports window-based and rate-based congestion schemes. Window-based schemes use congestion windows at both sender and receiver sides, where the size of congestion windows is adjustable based on congestion state. On the other hand, rate-based schemes are established based on feedback algorithms and accept sending rate based on receiver side only [21].

In [22], Distributed Fair Power Adjustment scheme for VANETs (D-FPAV) was introduced, which proposes a dynamic control strategy for congestion control within transmission range. D-FPAV short beacon messages broad-casted periodically and provide vehicles position, direction and speed. In this scheme, receiving beacons from long range transmission are very low, while bandwidth is poorly managed due to greater beaconing and high traffic density.

An metaheuristic-based approach called Uni-Objective tabu search (UOTabu) is proposed in [19] to handle VANETs congestion, which offers performing calculations to determine channel usage level along with transmission range, which consequently leads to rate-tuning to minimise delay. This uni-objective scheme considers delay as an objective function. The study provides a comparison between CSMA/CA, D-FPAV and UOTabu schemes and suggests that UOTabu help decrease delay and increase throughput with the expense of higher computational complexities. The proposed scheme in this study does not consider the impact of emergency message broadcast on overall network performance and is verified with small size problems experimented only for time periods of 3 seconds.

Significance of studies can easily be judged based on above-mentioned literature, researchers implemented proactive scheduling based on future prediction of traffic as explained in [12], scheduling based on prediction is based on some probability of occurrence which effect QoS, while tabu search-based scheduling [19] implemented to address the delay, but the experimented time window is only 
3 seconds, which does not allow the network to be congested with chosen problem sizes. Under such circumstances, tabu search would only incur more complexity overhead rather than help solve the congestion problem. On the other hand, the expected benefit of tabu search can only be realised in the cases of heavy-loaded networks running at least 1 hour time.

\section{Route Optimization using Tabu Search}

In this study, we decided to tackle the problem of heavily-loaded VANETS experimented for enduring much longer time periods using a bespoke tabu search approach. The implemented tabu search approach is to address congestion in VANETs considering both Urban and Highway environments. The main functionality expected of tabu search algorithm to help find optimal route, which resolves congestion problem of vehicular communication. This sort of dynamic routing/scheduling problems require rather instant and cannot afford long-elapsing decision processes. Hence, well-engineered decision processes such as local-search algorithms can be useful in searching for the best solution within such short time windows. Given these circumstances, existing state-of-art neighbourhood and local search approaches can be seen as candidates to solve these dynamic problems, however, there is a substantial risk of providing suboptimal solutions from the regions under consideration.

Tabu Search [3,13] is one of most mature metaheuristic approaches wellknown with proven success [5], which offers facilitating local search rules in such a way that the best of neighbourhood is replaced with the current solution regardless of whether the solution is better or worse than the current one. As long as the best of the process is recorded, tabu search helps change the search regions systematically in this way. Moreover applied and issued moves within the search space are frozen for not to be re-considered for a particular while in order to prevent the search process from falling in vicious circle. The implemented algorithm is using memory structures to manage the visited solutions and if the potential solution has been previously visited within the short period of time or has violated a rule then it will be marked as tabu, then the algorithm will not consider this possibility repeatedly. The performance of proposed scheme is measured by calculating average delay, throughput, message generation rate and packet loss. Performance analysis is performed to compare it with other state of the art schemes.

This approach is implemented to comprise of two parts. The first one is the stage of priority allocation and the other is the stage of message scheduling. Priority allocation stage allocates priorities to the messages as in Fig. 2. As is shown, the complete mechanism works from application layer to physical layer. In VANETs, Global Positing System (GPS) provides velocity, direction and location to the applications and then all the data moves to network layer. It is also part of the responsibilities of application layer to send broadcast messages to networks, which can include information, guidance and suggestions about routes such as information about upcoming congestion and suggestions according to that, average travel time to destination etc. Incoming messages can be 


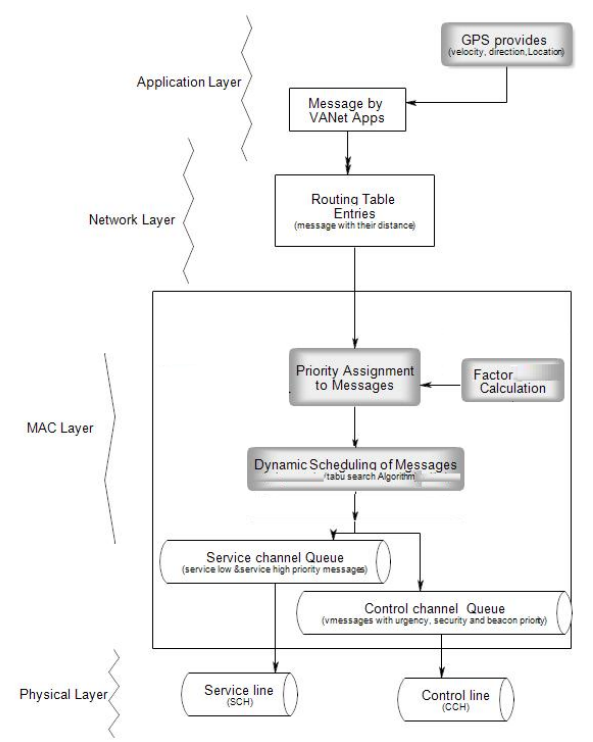

Fig. 2: Scheduling Procedure using Tabu Search with message priorities

of broadcast, uni-cast, or multi-cast. Routing table entries are created which shows message with their distances. In Medium Access Controller (MAC) layer, priorities are allocated to incoming messages based on calculated factors emerging last second. tabu search algorithm transfer data to Service channel queue which include service low and high priority messages and to control channel queue which categories messages based on urgency, security and beacon priority. Finally messages coming from Service channel queue move to service line $(\mathrm{SCH})$ and message from control channel queues move to Control line $(\mathrm{CCH})$ at physical layer.

Tabu search algorithm implemented in this study starts with initialising a number of parameters; message type, factors < delays, congestion, intersection >, the maximum number of iteration Tabu List and all counts including intensification. The algorithm is set to run until the pre-set stopping criterion is met.

A typical problem state is a set of messages in the queue, denoted as $\mathcal{M}$, ordered based on associated priorities, $\mathcal{P}$. Hence, a set of messages to be transmitted can be denoted as $X=\left\{x_{i}|i=0, . .,| \mathcal{M} \mid\right\}$, where $x_{i}$ denotes a typical message representing a pair of $\left\langle m_{i}, p_{i}>\right.$ and $m_{i}$ and $p_{i}$ denote $i_{t h}$ message and its priority, respectively. A randomly generated solution will have an order based on the priorities. A neighbourhood function $f_{N}\left(X_{i}\right)$ takes an existing solution and randomly swaps any two messages, say $x_{j}$ with $x_{l}$, if their priorities are equal or very close to each other, iff $p_{j}=p_{l}$ OR $\left|p_{j}-p_{l}\right| \leq \Theta$, where $\Theta$ is a threshold setup for differentiating priorities. A set of neighbours, $N=\left\{n_{k}|k=0, . .| N \mid,\right\}$, is generated using $f_{N}($.$) to the pre-set neighbourhood size. A candidate set,$ $C=\left\{c_{j}|j=0, . .| C \mid,\right\}$, is filtered out of $N$ with checking if any neighbour, $n_{k}$, is resulted of any tabu move. In each iteration, the best performing $c_{j}$ is replaces the current solution, $X_{i} \rightarrow c_{j}$. 
Once a neighbouring solution, $n_{k}$, is generated, the action taken, which is called move and denoted with $\left.m v<x_{i}, x_{j}\right\rangle$, is recorded in the short memory. Once $X_{i}$ is replaced with $c_{j}$, then $m v<X_{i}, c_{j}>$ will be added to the tabu list, $T=\left\{t_{m}<X_{m}, c_{m}>|m=0, . .| T \mid,\right\}$, which is aged iteration-by-iteration, and released once iterated $|t|$ times. Tabu list is considered as the long-term memory in this optimisation process. The quality of each solution is measured through network simulations, which are selected to be average packet delay and average packet loss. This calculation is conducted once a particular state of the problem (solution) is passed into the network simulation in an opted data structure.

Following initialisation step, a typical tabu search iteration consists of neighbourhood and candidate list generations, selecting the best candidate as the new solution and admitting the move in tabu list, and finally updating the tabu status of the whole tabu list. There might be some conflicting cases, where none of the solutions in the neighbourhood is eligible to be candidate since all are included in tabu list. Such cases would trigger re-generating the neighbourhood. If successively generated neighbourhoods are not producing eligible solutions within a pre-set intensification period, which is deviced to remedy this sort of cases.

\section{Performance Evaluation}

In order to evaluate performance of proposed approach in comparison with existing commonly used approaches, network and mobility simulators, SUMO and NS-3, are used. Connection between SUMO and NS-3 is defined using MOVE, which is a mode used for generating mobility in VANETs. As an urban scenario, a six-lane road within urban environment is simulated. Table. 1 presents the parametric details considered in the simulation, while $802.11 \mathrm{p}$ is taken into account as an interconnection standard. CSMA/CA approach is adopted as transferring approach in MAC layer, Nakagami and 2-dimensional grounds (models to show highway conditions) are preferred to design propagation into urban and highway conditions. The data traffic is assumed to conform the Poisson distribution.

Table 1: Parameters to configure simulation for highway* and urban** cases

\begin{tabular}{|l|l|l|l|}
\hline Parameters & Value & Parameters & Value \\
\hline MAC type & $802.11 \mathrm{p}$ & Bandwidth & $75 \mathrm{MHz}$ \\
\hline Frequency & $5.850 \mathrm{GHz}$ to 5.925 & Simulation time & $200 \mathrm{sec}$ to $1 \mathrm{hour}$ \\
\hline Message Size & $\begin{array}{l}\text { Emergency }(578 \text { bytes }) \\
\text { beacon }(500 \text { bytes })\end{array}$ & Speed of Vehicles & $\begin{array}{l}80-120 \mathrm{~km} / \mathrm{h}^{*} \\
0-50 \mathrm{~km} / \mathrm{h}^{* *}\end{array}$ \\
\hline Number of Lanes & $\begin{array}{l}4(2 \text { in every position) } \\
6(3)^{* *}\end{array}$ & Total road length & $\begin{array}{l}5400 \mathrm{~m}^{*} \\
1600 \mathrm{~m}^{*} \times 1700 \mathrm{~m}^{* *}\end{array}$ \\
\hline Number of vehicles & $400,300,200,150,100,50$ & Transmission rate & $10 \mathrm{Mbps}$ \\
\hline
\end{tabular}

\subsection{Simulation parameters and environments}

Performance of suggested approaches are examined with the state-of-the-art approaches known as Enhanced Distributed Channel Access(EDCA), First In First Out (FIFO) and dynamic scheduling. Across these performance evaluations, the following key performance indicators (KPI) are among those to be considered for examination purposes:- 
- Message lost count during simulation run time, the quantity of messages lost is checked to find the ratio of loss.

- Average delay in transmission from dispatcher to receiver, average time needed for transferring messages.

- Ratio of message loss as the quantity of messages lost in comparison with no of transferred messages.

- Average throughput within unit of time, which is the ratio of efficiently received messages on interconnection channels.

- Waiting time in queue, before de-queuing and transmitting towards channels, average waiting time for messages in $\mathrm{SCH}$ and $\mathrm{CCH}$ queues.

As may be seen, two main performance areas are identified; delay and packet loss, which have been chosen as the two main KPIs in this study. The following evaluations and discussions have been put together on the basis of these two in averaged form. All the calculations are conducted to determine these two KPI values through specifically configured network simulation with the parametric details provided in Table.1.

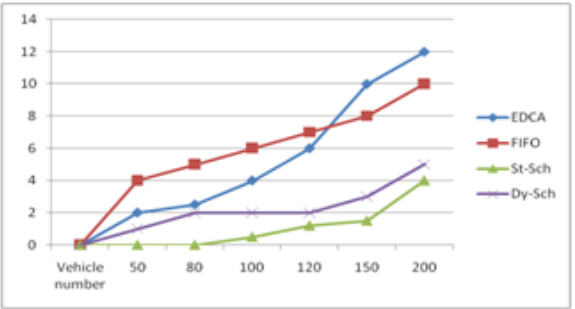

(a) average delay

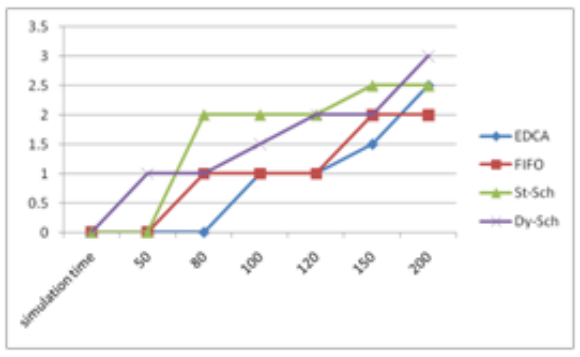

(c) average throughput

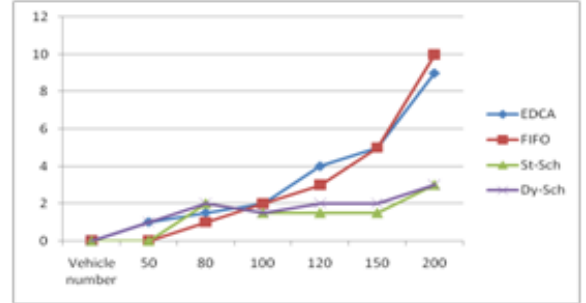

(b) packet loss

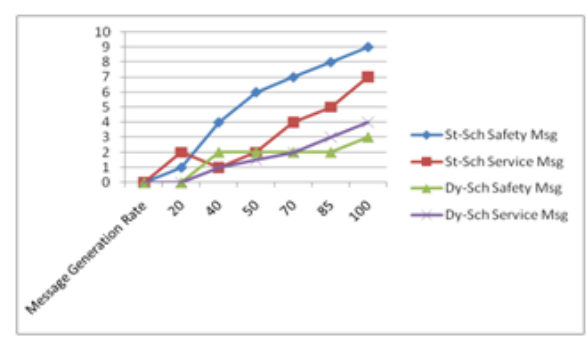

(d) message generation rate

Fig. 3: Performances in highway environments

\subsection{Comparative Analysis of Proposed Algorithms with others}

As per comparative analysis, tabu search-based scheduling (St-Sch) approach, which will be named tabu Scheduling here-forth, is observed in general that it helps reduce the delay of transmission while FIFO does not work effectively in high quality transport environments since it de-queues the packets with none prioritised ones. EDCA prioritisation mechanism fails to manage congestion when 
there are large numbers of priority messages that escalates to high delay for low priority data.

As indicated in Fig. 3a the average delay in cases of highways by the proposed scheme is $4 \mathrm{~ms}$ when there are 200 vehicles, while the best of the state of the art techniques considered, so-called dynamic scheduling, produces much higher delay starting form 5.5ms. On the other hand, FIFO and EDCA mechanisms are much less efficient as delay increases exponentially with growing number of vehicles. Fig. 3b shows the packet loss ratio on different load of vehicles. Studies suggest that dynamic scheduling (Dy-Sch) and tabu scheduling (St-Sch) methods perform better than other rival methods.

Performance analysis is extended with cases in urban environment alongside the highway cases. The results are plotted as in Fig. 4a and Fig. 4b, where it is clearly observed that EDCA and FIFO provide the worst performance with growing number of vehicles, especially beyond 120, while both dynamic scheduling and the proposed approach, tabu scheduling, perform very similarly noting that the measure of average packet loss by tabu and dynamic scheduling approaches are much less, in some cases the performance of dynamic scheduling is little bit higher.

Fig. 3c suggests that the average throughput by tabu scheduling is higher and offer high stability in comparison to rival approaches, similarly while comparing safety and services message with tabu and dynamic search, it is clearly shown in Fig. 3d that message generation rate of tabu scheduling is much higher assuring a higher level of security and efficient processing of service messages.

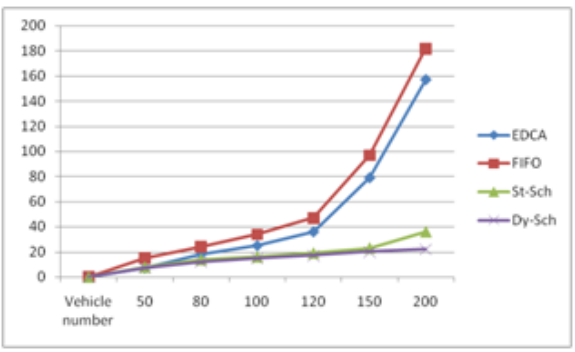

(a) average delay

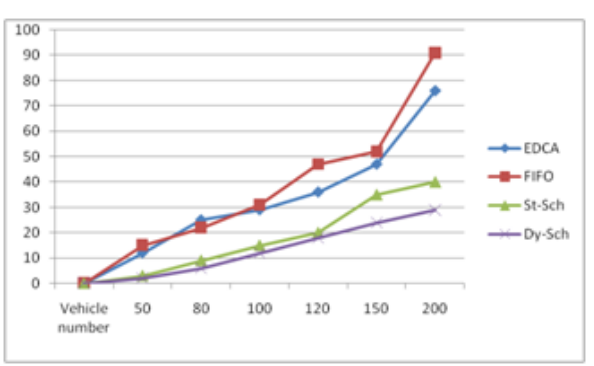

(b) average packet lost

Fig. 4: Performances in urban environment in vehicle count

Table 2: Average delay in urban and highway environments with growing number of vehicles

\begin{tabular}{c|rrrr|rrrc}
\hline $\begin{array}{c}\text { Number of } \\
\text { Vehicles }\end{array}$ & \multicolumn{4}{|c|}{ Urban } & \multicolumn{4}{c}{ Highway } \\
\hline 100 & EDCA & FIFO Dy-Sch & St-Sch & EDCA & FIFO Dy-Sch & St-Sch \\
200 & 157 & 182 & 25 & 22 & 23 & 6 & 2 & 0.5 \\
300 & 300 & 510 & 48 & 31 & 30 & 25 & 10 & 7 \\
400 & 501 & 780 & 80 & 39 & 50 & 40 & 19 & 10 \\
\hline
\end{tabular}


Table. 2 presents the average delay scores in transmission by each scheme, comparatively, in high load circumstances, the performance of the schemes are measured with different loads from 100 to 400 vehicles in cases of both urban and highway infrastructures simulated for 1 hour. It can be observed that the average delay in urban cases are much higher than highways, as expected. It is also clear that both dynamic scheduling and tabu scheduling schemes significantly help reduce the average delay beyond 300 vehicles, in both environmental circumstances, while tabu scheduling clearly achieves better than dynamic scheduling. Average delay caused by tabu scheduling remains as 31 in urban and 7 in highway environments when the network size is 300 vehicles, it become 39 and 10 when the network is of 400 vehicles.

Table 3: Average packet-loss in urban and highway environments with growing number of vehicles

\begin{tabular}{c|rrcc|rccc}
\hline $\begin{array}{c}\text { Number of } \\
\text { Vehicles }\end{array}$ & \multicolumn{4}{|c|}{ Urban } & \multicolumn{4}{c}{ Highway } \\
\hline 100 & 29 & 31 & 12 & 9 & 2 & 2 & 1.5 & 1.5 \\
200 & 76 & 91 & 39 & 18 & 9 & 10 & 3 & 3 \\
300 & 136 & 216 & 56 & 25 & 17 & 23 & 10 & 5 \\
400 & 229 & 481 & 86 & 31 & 39 & 49 & 19 & 8.5 \\
\hline
\end{tabular}

Table. 3 shows average packet loss score by each scheme comparatively, where packet loss measure is much lower in highway circumstances as expected. The network's workload is set to high level, as is in delay cases, spanning from 100 to 400 vehicles. It is observed that both dynamic and tabu scheduling schemes remain significantly lower than the other two classical schemes, especially beyond 300 vehicles. Here, the score of tabu is 25 losses in urban and 5 in highway environments under the circumstances of the network of 300 vehicles, while 31 and 8.5 achieved for a network of 400 vehicles. This clarifies and confirms the usefulness of tabu search in solving these problems.

\section{Conclusion}

In this paper, a novel routing/scheduling approach called tabu scheduling is studied for handling congestion control and message traffic in highly dynamic networks such as Vehicle Ad-hoc Networks (VANET). The proposed technique is experimented and evaluated in comparison with two classical (FIFO, EDCA) and one most recently introduced state-of the-art approaches. It is observed that the proposed approach helps handle congestion much more efficiently for both urban and highway environments. Tabu scheduling results are highly admirable as it focuses on transmission delay and message superiorities, on the other hand, dynamic approach is only focusing on superiorities messages. Results show that tabu Scheduling rank first than dynamic scheduling while EDCA and FIFO are least efficient. Thus, within VANETs, reliable and secure atmosphere provided through tabu scheduling. 


\section{References}

1. Golestan, K., Jundi, A., Nassar, L., Sattar, F., Karray, F., Kamel, M., and Boumaiza, S. (2012). Vehicular ad-hoc networks (VANETs): capabilities, challenges in information gathering and data fusion. In Autonomous and Intelligent Systems (pp. 34-41). Springer Berlin Heidelberg.

2. Golestan, K., Sattar, F., Karray, F., Kamel, M., and Seifzadeh, S. (2015). Localization in vehicular ad hoc networks using data fusion and V2V communication. Computer Communications, 71, 61-72.

3. Barbarosoglu, G. and Ozgur, D. (1999). A tabu search algorithm for the vehicle routing problem. Computers \& Operations Research, 26(3), pp. 255-270.

4. Ghosh, T., and Mitra, S. (2012, November). Congestion control by dynamic sharing of bandwidth among vehicles in VANET. In Intelligent Systems Design and Applications (ISDA), 2012 12th International Conference on (pp. 291-296). IEEE.

5. C. H. Guan, Y. Cao and J. Shi, (2010). Tabu Search Algorithm for Solving the Vehicle Routing Problem. Third International Symposium on Information Processing, Qingdao, 2010, pp. 74-77.

6. Sepulcre, M., Gozalvez, J., Altintas, O., and Kremo, H. (2016). Integration of congestion and awareness control in vehicular networks. Ad Hoc Networks, 37, 29-43.

7. Singh, M., Rathod, D., and Upadhyay, J. (2017). Variants of Scheduling in Distributed Networking using Vehicular Ad-hoc Networks, IJIRST, National Conference on Latest Trends in Networking and Cyber Security, March 2017, pp. 84-89.

8. Sepulcre, M., Gozalvez, J., Altintas, O., and Kremo, H. (2016). Integration of congestion and awareness control in vehicular networks. Ad Hoc Networks, 37, $29-43$.

9. Taherkhani, N., and Pierre, S. (2012, October). Congestion control in vehicular ad hoc networks using meta-heuristic techniques. In Proceedings of The 2nd ACM International Symposium on Design and Analysis of Intelligent Vehicular Networks and Applications (pp. 47-54). ACM.

10. Djahel, S., and Ghamri-Doudane, Y. (2012, April). A robust congestion control scheme for fast and reliable dissemination of safety messages in VANETs. In Wireless Communications and Networking Conference (WCNC), 2012 IEEE (pp. 22642269). IEEE.

11. Ghosh, A., Paranthaman, V. V., Mapp, G., Gemikonakli, O., and Loo, J. (2015). Enabling seamless V2I communications: toward developing cooperative automotive applications in VANET systems. IEEE Communications Magazine, 53(12), 80-86.

12. Hannk, G., (2013). Congestion and awareness control in cooperative vehicular networks, In: Mobile communications seminar SS 2013.

13. Ho, S.C., and Haugland, D., (2004). A tabu search heuristic for the vehicle routing problem with time windows and split deliveries. Computers \& Operations Research, 31(12), pp. 1947 - 1964.

14. Y. Zang, L. Stibor, X. Cheng, H.-J. Reumerman, A. Paruzel, A. Barroso, Congestion control in wireless networks for vehicular safety applications, In: Proceedings of the 8th European wireless conference, 2007.

15. R. Baldessari, D. Scanferla, L. Le, W. Zhang, A. Festag, Joining forces for vanets: a combined transmit power and rate control algorithm, In: 7th international workshop on intelligent transportation (WIT), Hamberg, Germany, 2010, pp. 15.

16. B.M. Mughal, A.A. Wagan, H. Hasbullah, Efficient congestion control in VANET for safety messaging, In: Information technology (ITSim), 2010 international symposium in, Kuala Lumpur, 2010, pp. 654659 
17. M.S. Bouassida, M. Shawky, A cooperative congestion control approach within VANETs: formal verification and performance evaluation, EURASIP Journal of Wireless Communication 2010 (2010) 11.

18. L. Le, R. Baldessari, P. Salvador, A. Festag, W. Zhang, Performance evaluation of beacon congestion control algorithms for VANETs, In: Global telecommunications conference (GLOBECOM 2011), Houston, TX, USA, 2011 IEEE, 2011, pp. 16.

19. Taherkhani, N., and Pierre, S. (2012, October). Congestion control in vehicular ad hoc networks using meta-heuristic techniques. In Proceedings of The 2nd ACM International Symposium on Design and Analysis of Intelligent Vehicular Networks and Applications (pp. 47-54). ACM.

20. I. Bratko, R.S. Michalski, M. Kubat, Machine learning and data mining: methods and applications, 1999.

21. B. Subramani, E. Chandra, A survey on congestion control, Global J Comput Sci Technol 9 (2010).

22. M. Torrent-Moreno, P. Santi, H. Hartenstein, Distributed fair transmit power adjustment for vehicular ad hoc networks, In: Sensor and ad hoc communications and networks, 2006. SECON'06. 2006 3rd annual IEEE communications society on, Reston, VA, 2006, pp. 479488.

23. J. He, H.-H. Chen, T.M. Chen, W. Cheng, Adaptive congestion control for DSRC vehicle networks, IEEE Commun Lett 14 (2010) 127129.

24. Lu, N., Zhang, N., Cheng, N., Shen, X., Mark, J.W. and Bai, F., 2013. Vehicles meet infrastructure: Toward capacitycost tradeoffs for vehicular access networks. IEEE transactions on intelligent transportation systems, 14(3), pp.1266-1277. 